\title{
RESOLVING SYSTEMS OF NONLINEAR INTEGRAL EQUATIONS VIA C-CLASS FUNCTIONS
}

\author{
LJILJANA PAUNOVIĆ ${ }^{1}$ \\ ${ }^{1}$ Teacher Education School in Prizren-Leposavić, University of Priština, Leposavić, Serbia.
}

\begin{abstract}
Fixed point theorems for monotone operators in ordered metric spaces are widely investigated and have found various applications in differential and integral equations, see Nieto \& Rodríguez-López (2005); Nieto \& Rodríguez-López (2007); Wu (2008). Motivated by the work in Agarwal et al. (2012); Luong \& Thuan (2011) we study the existence of solutions for a system of nonlinear integral equations in ordered b-metric spaces using the results given in this paper.
\end{abstract}

Key words: b-metric spaces, compatible mappings, P-increasing, integral equation, C-class functions.

\section{INTRODUCTION}

In Guo \& Lakshmikantham (1987) studied the concept of coupled fixed points. Later, in Bhaskar \& Lakshmikantham (2006) studied monotone property and supported this by providing an application to the existence of periodic boundary value problems.

In Bakhtin (1989) introduced the concept of a b-metric space as a generalization of metric spaces. In Czerwik (1993) and Czerwik (1998) extended many results related to the bmetric spaces. Since then, several papers have been published on the fixed point theory of various classes of single-valued and multi-valued operators in b-metric spaces (Akkouchi, 2011; Aydi et al., 2012; Boriceanu, 2009; Boriceanu et al., 2010; Bota et al., 2011; Hussain \& Shah, 2011; Olatinwo, 2008; Huang et al., 2015; Mustafa et al., 2013; Mustafa et al., 2014; Ansari et al., 2014; Aghajani et al., 2014).

In this paper, we use the notion of C-class of function which is generalization of altering distance function and by using this definition we have improved the results of Hussain, Abbas, Azam and Ahmad (Hussain et al., 2014) in the setting of bmetric space. Also we provide and an application to an integral equation to support our results presented here.

Consistent with Bakhtin (1989); Czerwik (1993); Czerwik (1998); Huang et al. (2015) the following definitions and results will be needed in the sequel.

Definition 1.1. (Bakhtin, 1989; Czerwik, 1993) Let $M$ be a nonempty set and $B \geq 1$ be a given real number. A function $d_{b}: M \times M \rightarrow R^{+}$is a b-metric if the following conditions are satisfied:

(b1) $d_{b}(\bar{x}, \bar{y})=0$ if and only if $\bar{x}=\bar{y}$;

(b2) $d_{b}(\bar{x}, \bar{y})=d_{b}(\bar{y}, \bar{x})$;

(b3) $d_{b}(\bar{x}, \bar{z}) \leq B\left[d_{b}(\bar{x}, \bar{y})+d_{b}(\bar{y}, \bar{z})\right]$

\footnotetext{
* Corresponding author: 1jiljana.paunovic76@gmail.com
} MATHEMATICS for all $\bar{x}, \bar{y}, \bar{z} \in M$.

The pair $\left(M, d_{b}\right)$ is called a b-metric spaces. It is well know that each metric space is also b-metric (with $B=1$ ) while the converse is not true.

Definition 1.2. Let $M$ be a nonempty set. Then $\left(M, \leq, d_{b}\right)$ is called a partially ordered b-metric space if and only if $d_{b}$ is a bmetric on a partially ordered set $(M, \leq)$.

Definition 1.3. (Boriceanu et al., 2010) Let $\left(M, d_{b}\right)$ be a bmetric space. Then a sequence $\left\{x_{p}\right\}$ in $M$ is called bconvergent if and only if there exists $\bar{x} \in M$ such that $d_{b}\left(x_{p}, \bar{x}\right) \rightarrow 0 \quad$ as $\quad p \rightarrow+\infty$. In this case, we write $\lim _{p \rightarrow \infty} x_{p}=\bar{x}$.

Definition 1.4. (Boriceanu et al., 2010) Let $\left(M, d_{b}\right)$ be a bmetric space. Then a sequence $\left\{x_{p}\right\}$ in $M$ is called b-Cauchy if and only if $d_{b}\left(x_{p}, x_{q}\right) \rightarrow 0$, as $p, q \rightarrow+\infty$.

Definition 1.5. (Boriceanu et al., 2010) The b-metric space $\left(M, d_{b}\right)$ is b-complete if every b-Cauchy sequence in $M$ bconverges.

In Ansari et. al. (2014) introduced the concept of C-class functions which cover a large class of contractive conditions.

Definition 1.6. (Ansari et al., 2014) A mapping $W:[0, \infty)^{2} \rightarrow R$ is called C-class function if it is continuous and satisfies following axioms:

(1) $W(\bar{s}, \bar{t}) \leq \bar{s}$;

(2) $W(\bar{s}, \bar{t})=\bar{s}$ implies that either $\bar{s}=0$ or $\bar{t}=0$ for all $\bar{s}, \bar{t} \in[0, \infty)$.

We denote C-class functions as $C$. 
Definition 1.7. ((Hussain et al., 2014), Definition 7) Suppose that $H, P: M \times M \rightarrow M$ are two mappings. Then $H$ is said to be $P$ - increasing with respect to $\leq$ if for all $\bar{x}, \bar{y}, \xi, g \in M$ with $P(\bar{x}, \bar{y}) \leq P(\xi, g)$ we have $H(\bar{x}, \bar{y}) \leq H(\xi, g)$.

Definition 1.8. ((Hussain et al., 2014), Definition 10) An element $(\bar{x}, \bar{y}) \in M \times M$ is called a coupled coincidence point of mappings $H, P: M \times M \rightarrow M \quad$ if $H(\bar{x}, \bar{y})=P(\bar{x}, \bar{y}) \quad$ and $H(\bar{y}, \bar{x})=P(\bar{y}, \bar{x})$.

Definition 1.9. ((Hussain et al., 2014), Definition 12) Let $H, P: M \times M \rightarrow M$. We say that the pair $\{H, P\}$ is generalized compatible if

$$
\left\{\begin{array}{l}
d_{b}\left(H\left(P\left(x_{p}, y_{p}\right), P\left(y_{p}, x_{p}\right)\right), P\left(H\left(x_{p}, y_{p}\right), H\left(y_{p}, x_{p}\right)\right)\right) \\
\rightarrow 0 \text { as } p \rightarrow \infty \\
d_{b}\left(H\left(P\left(y_{p}, x_{p}\right), P\left(x_{p}, y_{p}\right)\right), P\left(H\left(y_{p}, x_{p}\right), H\left(x_{p}, y_{p}\right)\right)\right) \\
\rightarrow 0 \text { as } p \rightarrow \infty
\end{array}\right.
$$

whenever $\left(x_{p}\right)$ and $\left(y_{p}\right)$ are sequences in $M$ such that

$$
\left\{\begin{array}{l}
\lim _{p \rightarrow \infty} H\left(x_{p}, y_{p}\right)=\lim _{p \rightarrow \infty} P\left(x_{p}, y_{p}\right)=t_{1} \\
\lim _{p \rightarrow \infty} H\left(y_{p}, x_{p}\right)=\lim _{p \rightarrow \infty} P\left(y_{p}, x_{p}\right)=t_{2} .
\end{array}\right.
$$

Definition 1.10. (Bhaskar \& Lakshmikantham, 2006) Let $(M, \leq)$ be a partially ordered set. Then the mapping $H: M \times M \rightarrow M$ is said to have the mixed monotone property if $H$ is monotone non-decreasing in its first argument and is monotone non-increasing in its second argument, that is, for all $x_{1}, x_{2} \in M, x_{1} \leq x_{2}$ implies $H\left(x_{1}, \bar{y}\right) \leq H\left(x_{2}, \bar{y}\right)$, for any $\bar{y} \in M \quad$ and for all $y_{1}, y_{2} \in M, y_{1} \leq y_{2} \quad$ implies $H\left(\bar{x}, y_{1}\right) \geq H\left(\bar{x}, y_{2}\right)$, for any $\bar{x} \in M$.

In the paper Hussain et al. (2014), in Theorem 15, it is shown the example when $(M, \leq)$ us a partially ordered set such that there exists a complete metric $d$ on $M$.

Therefore, in this paper we gives Theorem 1.11 which represents the continuation of Theorem 15 from Hussain et al. (2014) on b-metric spaces whit the implementation of the functions of the C-class. However, in our case, in the following Theorem 1.11 the condition (1) highly differs from the condition in Theorem 15 (Hussain et al., 2014).

Theorem 1.11. Let $\left(M, \leq, d_{b}\right)$ be an ordered complete b-metric space (with parameter $B>1$ ). Assume that $H, P: M \times M \rightarrow M$ are two generalized compatible mappings such that $H$ is $P$ increasing with respect to $\leq, P$ is continuous and has the mixed monotone property, and there exist two elements $x_{0}, y_{0} \in M$ with

$P\left(x_{0}, y_{0}\right) \leq H\left(x_{0}, y_{0}\right)$ and $P\left(y_{0}, x_{0}\right) \geq H\left(y_{0}, x_{0}\right)$.

Suppose that there exist $\varphi \in \Phi$ and $\psi \in \Psi, W$ is C-class such that

$$
\begin{gathered}
\varphi\left(\bar{s}^{a} d_{b}(H(\bar{x}, \bar{y}), H(\xi, g))\right) \\
\quad \leq W\left(\varphi\left(\frac{d_{b}(P(\bar{x}, \bar{y}), P(\xi, g))+d_{b}(P(\bar{y}, \bar{x}), P(g, \xi))}{2}\right)\right. \\
\left.\quad \psi\left(\frac{d_{b}(P(\bar{x}, \bar{y}), P(\xi, g))+d_{b}(P(\bar{y}, \bar{x}), P(g, \xi))}{2}\right)\right)
\end{gathered}
$$

for all $\bar{x}, \bar{y}, \xi, g \in M, \quad a>1 \quad$ with $P(\bar{x}, \bar{y}) \leq P(\xi, g)$ and $P(\bar{y}, \bar{x}) \geq P(g, \xi)$. Suppose that for any $\bar{x}, \bar{y} \in M$, there exist $\xi, g \in M$, such that

$\left\{\begin{array}{l}H(\bar{x}, \bar{y})=P(\xi, g) \\ H(\bar{y}, \bar{x})=P(g, \xi) .\end{array}\right.$

Also suppose that either

(a) $H$ is continuous or

(b) $M$ has the following property

(i) If a non-decreasing sequence $\left\{x_{p}\right\} \rightarrow \bar{x}$, then $x_{p} \leq \bar{x}$ for all $p$

(ii) If a non-increasing sequence $\left\{y_{p}\right\} \rightarrow \bar{y}$, then $\bar{y} \leq y_{p}$ for all $p$.

Then, $H$ and $P$ have a coupled coincidence point in $M$. The proof of this theorem follows directly.

Hussain, Abbas, Azam and Ahmad in Hussain et al. (2014) in Corollary 22 gives the situation when $(M, \leq)$ us a partially ordered set and suppose there is a metric $d$ on $M$ such that $(M, d)$ is a complete metric space.

In this paper we gives Corollary 1.12 that shows the broadening of Corollary 22 in Hussain et al. (2014) on b-metric spaces by using the C-class functions. It should be noted that in our example, in Corollary 1.12 which follows the condition (2) there is a great difference from the condition in Corollary 22 in Hussain et al. (2014).

Corollary 1.12. Let $\left(M, \leq, d_{b}\right)$ be an ordered complete b-metric space (with parameter $B>1$ ) and suppose there is a metric $d_{b}$ on $M$ such that $\left(M, d_{b}\right)$ is a complete metric space. Assume that $H: M \times M \rightarrow M$ be an increasing map with respect to $\leq$ and there exist two elements $x_{0}, y_{0} \in M$ with $x_{0} \leq H\left(x_{0}, y_{0}\right)$ and $y_{0} \geq H\left(y_{0}, x_{0}\right)$. 
Suppose there exists $\psi \in \Psi, W$ is C-class such that

$$
\begin{aligned}
& \bar{s}^{a}\left(d_{b}(H(\bar{x}, \bar{y}), H(\xi, g))\right) \\
& \leq W\left(\frac{d_{b}(\bar{x}, \xi)+d_{b}(\bar{y}, g)}{2},\right. \\
& \left.\psi\left(\frac{d_{b}(\bar{x}, \xi)+d_{b}(\bar{y}, g)}{2}\right)\right)
\end{aligned}
$$

for all $\bar{x}, \bar{y}, \xi, g \in M, a>11$ with $\bar{x} \leq \xi$ and $\bar{y} \geq g$. Also suppose that either

(a) $H$ is continuous or

(b) $M$ has the following property

(i) If a non-decreasing sequence $\left\{x_{p}\right\} \rightarrow \bar{x}$, then $x_{p} \leq \bar{x}$ for all $p$

(ii) If a non-increasing sequence $\left\{y_{p}\right\} \rightarrow \bar{y}$, then $\bar{y} \leq y_{p}$ for all $p$.

Then $H$ has a coupled fixed point.

\section{RESULTS}

Fixed point theorems for monotone operators in ordered metric spaces are widely investigated and have found various applications in differential and integral equations see Nieto \& Rodríguez-López (2005); Nieto \& Rodríguez-López (2007); Wu 2008)). Motivated by the work in Agarwal et al. (2012); Luong $\&$ Thuan (2011) we study the existence of solutions for a system of nonlinear inregral equations using the results given in the previous section.

Let $E$ denote the class of those functions $\varepsilon:[0, \infty) \rightarrow[0, \infty)$ which satisfies the following conditions:

$\left(a_{1}\right) \quad \varepsilon$ is increasing

$\left(b_{1}\right) \quad$ there exists $\psi \in \Psi, W \in C$ such that

$$
\varepsilon(\tau)=\frac{1}{2 m} W\left(\frac{\tau}{2}, \psi\left(\frac{\tau}{2}\right)\right) \text { for all } \tau \in[0, \infty)
$$

Consider the integral equation

$$
\begin{aligned}
\bar{x}(\tau) & =\int_{a}^{b}\left(K_{1}(\tau, \phi)+K_{2}(\tau, \phi)\right)(h(\phi, \bar{x}(\phi))+l(\phi, \bar{x}(\phi))) d \phi \\
& +k(\tau)
\end{aligned}
$$

for all $\tau \in[a, b]$. We suppose that $K_{1}, K_{2}, h$ and $l$ satisfy the following conditions:

(i) $0 \leq K_{1}(\tau, \phi) ; 0 \leq K_{2}(\tau, \phi)$ for all $\tau, \phi \in[a, b]$;

(ii) There exist $\lambda, \mu>0$ and $\varepsilon \in \mathrm{E}$ such that for all $\bar{x}, \bar{y} \in R, \quad \bar{x} \geq \bar{y}$,

$0 \leq h(\tau, \bar{x})-h(\tau, \bar{y}) \leq \lambda \varepsilon(\bar{x}-\bar{y})$ and

$0 \leq l(\tau, \bar{x})-l(\tau, \bar{y}) \leq \mu \varepsilon(\bar{x}-\bar{y})$.

(iii) There is $\max \left\{\lambda^{2}, \mu^{2}\right\}\left\{\sup _{\tau \in[a, b]} \int_{a}^{b}\left(K_{1}(\tau, \phi)+K_{2}(\tau, \phi)\right) d \phi\right\}^{2} \leq m^{2}$,

for $m>0$.

(iv) There exist continuous functions $\bar{z}, \varpi:[a, b] \rightarrow R$ such as

$$
\bar{z}(\tau) \leq \int_{a}^{b} K_{1}(\tau, \phi)(h(\phi, \bar{z}(\phi))+l(\phi, \varpi(\phi))) d \phi
$$

$$
+\int_{a}^{b} K_{2}(\tau, \phi)(h(\phi, \varpi(\phi))+l(\phi, \bar{z}(\phi))) d \phi+k(\tau)
$$

and $\varpi(\tau) \geq \int_{a}^{b} K_{1}(\tau, \phi)(h(\phi, \varpi(\phi))+l(\phi, \bar{z}(\phi))) d \phi$

$+\int_{a}^{b} K_{2}(\tau, \phi)(h(\phi, \bar{z}(\phi))+l(\phi, \varpi(\phi))) d \phi+k(\tau)$

for all $\tau \in[a, b]$.

In Hussain et al. (2014), Theorem 26) we studied the integral equation (3) and proved that the mapping $H$ has a coupled fixed point that is a solution in $M=C([a, b], R)$.

Also, we overviewed the same integral equation (3) and proved that the mapping $H$ has a coupled fixed point that is a solution in $M=C([a, b], R)$, too, but in the range of b-metric spaces by the implementation of C-class functions. Our case gives different conditions $\left(b_{1}\right)$ and (iii) when compared to the conditions given in Theorem 26 in Hussain et al. (2014).

Theorem 2.1. Consider the integral equation (3) with $K_{1}, K_{2} \in C([a, b] \times[a, b], R), h, l \in C([a, b] \times R, R) \quad$ and $k \in C([a, b], R)$ and suppose that the conditions (i)-(iv) are satisfied. Then the integral equation (3) has a solution in $C([a, b], R)$.

Proof: The first part of proof of this theorem is very similar to the proof of Theorem 26 in Hussain et al. (2014). For the proof we use the following approach. Let $M=C([a, b], R)$ denote the space of all continuous functions defined on the interval $[a, b]$ to $R$. We endowed $M$ with the metric $d_{b}: M \times M \rightarrow R$ defined by

$d_{b}(\bar{x}, \bar{y})=\sup _{\tau \in[a, b]}|\bar{x}(\tau)-\bar{y}(\tau)|^{2}$, for all $\bar{x}, \bar{y} \in M$.

Compared to Theorem 26 in Hussain et al. (2014) where $(M, d)$ is a complete metric space and $(M, d, \leq)$ is a complete ordered metric space, in our case $\left(M, d_{b}\right)$ is a complete b-metric space and $\left(M, d_{b}, \leq\right)$ is a complete ordered b-metric space if $\bar{x} \leq \bar{y}$ whenever $\bar{x}(\tau) \leq \bar{y}(\tau)$ for all $\tau \in[a, b]$. Suppose $\left\{\xi_{p}\right\}$ 
is a monotone non-decreasing in $M$ that converges to $\xi \in M$. Then for every $\tau \in[a, b]$ the sequence of real numbers

$\xi_{1}(\tau) \leq \xi_{2}(\tau) \leq \ldots \leq \xi_{n}(\tau) \leq \ldots$

converges to $\xi(\tau)$. Therefore for all $\tau \in[a, b], p \in N$, $\xi_{p}(\tau) \leq \xi(\tau)$. Hence $\xi_{p} \leq \xi$ for all $p$. Similarly, we can verify that $\lim _{p} g(\tau)$ of a monotone non-increasing sequence $g_{p}(\tau)$ in $M$ is a lower bound for all the elements in the sequence. That is, $g \leq g_{p}$ for all $p$. Therefore, condition (b) of Corollary (1.12) holds.

Also, $\quad M \times M=C([a, b], R) \times C([a, b], R)$ is a partially ordered set if we define the following order relation on $M \times M$, for all $\bar{x}, \bar{y}, \xi, g \in M$, with $\bar{x} \leq \xi$ and $\bar{y} \geq g$.

A mapping $H: M \times M \rightarrow M$ define by

$H(\bar{x}, \bar{y})(\tau)=\int_{a}^{b} K_{1}(\tau, \phi)(h(\phi, \bar{x}(\phi))+l(\phi, \bar{y}(\phi))) d \phi$

$+\int_{a}^{b} K_{2}(\tau, \phi)(h(\phi, \bar{y}(\phi))+l(\phi, \bar{x}(\phi))) d \phi+k h(\tau)$ for all $\tau \in[a, b]$.

Now we will prove that $H$ is increasing. For $x_{1} \leq x_{2}$, that is $x_{1}(\tau) \leq x_{2}(\tau)$, for all $\tau \in[a, b]$, we have

$H\left(x_{1}, \bar{y}\right)(\tau)-H\left(x_{2}, \bar{y}\right)(\tau)$

$=\int_{a}^{b} K_{1}(\tau, \phi)\left(h\left(\phi, x_{1}(\phi)\right)+l(\phi, \bar{y}(\phi))\right) d \phi$

$+\int_{a}^{b} K_{2}(\tau, \phi)\left(h(\phi, \bar{y}(\phi))+l\left(\phi, x_{1}(\phi)\right)\right) d \phi+k(\tau)$

$-\int_{a}^{b} K_{1}(\tau, \phi)\left(h\left(\phi, x_{2}(\phi)\right)+l(\phi, \bar{y}(\phi))\right) d \phi$

$-\int_{a}^{b} K_{2}(\tau, \phi)\left(h(\phi, \bar{y}(\phi))+l\left(\phi, x_{2}(\phi)\right)\right) d \phi-k(\tau)$

$=\int_{a}^{b} K_{1}(\tau, \phi)\left(h\left(\phi, x_{1}(\phi)\right)-h\left(\phi, x_{2}(\phi)\right)\right) d \phi$

$+\int_{a}^{b} K_{2}(\tau, \phi)\left(l\left(\phi, x_{1}(\phi)\right)-l\left(\phi, x_{2}(\phi)\right)\right) d \phi \leq 0$.

Hence $H\left(x_{1}, \bar{y}\right)(\tau) \leq H\left(x_{2}, \bar{y}\right)(\tau)$ for all $\tau \in[a, b]$, that is $H\left(x_{1}, \bar{y}\right) \leq H\left(x_{2}, \bar{y}\right) . \quad$ Similarly, if $y_{1} \leq y_{2}$, that is $y_{1}(\tau) \leq y_{2}(\tau)$ for all $\tau \in[a, b]$, we have

$H\left(\bar{x}, y_{1}\right)(\tau)-H\left(\bar{x}, y_{2}\right)(\tau)$

$=\int_{a}^{b} K_{1}(\tau, \phi)\left(h(\phi, \bar{x}(\phi))+l\left(\phi, y_{1}(\phi)\right)\right) d \phi$

$+\int_{a}^{b} K_{2}(\tau, \phi)\left(h\left(\phi, y_{1}(\phi)\right)+l(\phi, \bar{x}(\phi))\right) d \phi+k(\tau)$ $-\int_{a}^{b} K_{1}(\tau, \phi)\left(h(\phi, \bar{x}(\phi))+l\left(\phi, y_{2}(\phi)\right)\right) d \phi$

$-\int_{a}^{b} K_{2}(\tau, \phi)\left(h\left(\phi, y_{2}(\phi)\right)+l(\phi, \bar{x}(\phi))\right) d \phi-k(\tau)$

$=\int_{a}^{b} K_{1}(\tau, \phi)\left(l\left(\phi, y_{1}(\phi)\right)-l\left(\phi, y_{2}(\phi)\right)\right) d \phi$

$+\int_{a}^{b} K_{2}(\tau, \phi)\left(h\left(\phi, y_{1}(\phi)\right)-h\left(\phi, y_{2}(\phi)\right)\right) d \phi \leq 0$.

Hence, $H\left(\bar{x}, y_{1}\right)(\tau) \leq H\left(\bar{x}, y_{2}\right)(\tau)$ for all $\tau \in[a, b]$, that is $H\left(\bar{x}, y_{1}\right) \leq H\left(\bar{x}, y_{2}\right)$. Thus $H(\bar{x}, \bar{y})$ is increasing. Now, for $\bar{x}, \bar{y}, \xi, g \in M$ such that $\bar{x} \leq \xi$ and $g \leq \bar{y}$, we conclude that

$d_{b}(H(\bar{x}, \bar{y})(\tau)-H(\xi, g)(\tau))$

$=\sup _{\tau \in[a, b]}|H(\bar{x}, \bar{y})(\tau)-H(\xi, g)(\tau)|^{2}$

$=\left\{\sup _{\tau \in[a, b]} \mid \int_{a}^{b} K_{1}(\tau, \phi)(h(\phi, \bar{x}(\phi))+l(\phi, \bar{y}(\phi))) d \phi\right.$

$+\int_{a}^{b} K_{2}(\tau, \phi)(h(\phi, \bar{y}(\phi))+l(\phi, \bar{x}(\phi))) d \phi+k(\tau)$

$\left.-\left(\begin{array}{l}\int_{a}^{b} K_{1}(\tau, \phi)(h(\phi, \xi(\phi))+l(\phi, g(\phi))) d \phi \\ +\int_{a}^{b} K_{2}(\tau, \phi)(h(\phi, g(\phi))+l(\phi, \xi(\phi))) d \phi+k(\tau)\end{array}\right) \mid\right\}^{2}$

$=\left\{\sup _{\tau \in[a, b]} \mid \int_{a}^{b} K_{1}(\tau, \phi)[(h(\phi, \bar{x}(\phi))-h(\phi, \xi(\phi)))\right.$

$+(l(\phi, \bar{y}(\phi))-l(\phi, g(\phi)))] d \phi$

$+\int_{a}^{b} K_{2}(\tau, \phi)[(h(\phi, \bar{y}(\phi))-h(\phi, g(\phi)))$

$+(l(\phi, \bar{x}(\phi))-l(\phi, \xi(\phi)))] d \phi \mid\}^{2}$

$\leq\left\{\sup _{\tau \in[a, b]} \mid \int_{a}^{b} K_{1}(\tau, \phi)\right.$

$\times[\lambda \varepsilon(\bar{x}(\phi)-\xi(\phi))+\mu \varepsilon(\bar{y}(\phi)-g(\phi))] d \phi+$

$\left.\int_{a}^{b} K_{2}(\tau, \phi)[\lambda \varepsilon(\bar{y}(\phi)-g(\phi))+\mu \varepsilon(\bar{x}(\phi)-\xi(\phi))] d \phi \mid\right\}^{2}$

$\leq \max \left\{\lambda^{2}, \mu^{2}\right\}\left\{\sup _{\tau \in[a, b]} \int_{a}^{b}\left[\left(K_{1}(\tau, \phi)+K_{2}(\tau, \phi)\right)\right]\right.$

$[\varepsilon|(\bar{x}(\phi)-\xi(\phi))|+\varepsilon|(\bar{y}(\phi)-g(\phi))|] d \phi\}^{2}$

As the function $\varepsilon$ is increasing and $\bar{y}(\tau) \geq g(\tau)$ for all $\tau \in[a, b]$, then

$\varepsilon(\bar{x}(\phi)-\xi(\phi)) \leq \varepsilon\left(d_{b}(\bar{x}, \xi)\right), \varepsilon(\bar{y}(\phi)-g(\phi)) \leq \varepsilon\left(d_{b}(\bar{y}, g)\right)$,

for all $\phi \in[a, b]$, we obtain 
$d_{b}(H(\bar{x}, \bar{y}), H(\xi, g))$

$\leq \max \left\{\lambda^{2}, \mu^{2}\right\}\left[\varepsilon^{2}\left(d_{b}(\bar{x}, \xi)\right)+\varepsilon^{2}\left(d_{b}(\bar{y}, g)\right)\right]$

$\times\left\{\sup _{\tau \in[a, b]} \int_{a}^{b}\left(K_{1}(\tau, \phi)+K_{2}(\tau, \phi)\right) d \phi\right\}^{2}$

$\leq m^{2}\left[\varepsilon^{2}\left(d_{b}(\bar{x}, \xi)\right)+\varepsilon^{2}\left(d_{b}(\bar{y}, g)\right)\right]$

$\leq m^{2} \varepsilon^{2}\left(d_{b}(\bar{x}, \xi)+d_{b}(\bar{y}, g)\right)$

$\leq \frac{m^{2}}{4 m^{2}} W\left(\frac{d_{b}(\bar{x}, \xi)+d_{b}(\bar{y}, g)}{2}, \psi\left(\frac{d_{b}(\bar{x}, \xi)+d_{b}(\bar{y}, g)}{2}\right)\right)$.

So, for $\bar{x} \leq \xi$ and $g \leq \bar{y}$, are following

$2^{2}\left(d_{b}(H(\bar{x}, \bar{y}), H(\xi, g))\right)$

$\leq W\left(\frac{d_{b}(\bar{x}, \xi)+d_{b}(\bar{y}, g)}{2}, \psi\left(\frac{d_{b}(\bar{x}, \xi)+d_{b}(\bar{y}, g)}{2}\right)\right)$.

Now by (iv) it follows that $\bar{z}(\tau) \leq H(\bar{z}, \varpi)(\tau)$ and $H(\varpi, \bar{z})(\tau) \leq \varpi(\tau)$ for all $\tau \in[a, b]$, that is $\bar{z} \leq H(\bar{z}, \varpi)$ and $H(\varpi, \bar{z}) \leq \varpi$. Thus all of the hypotheses of Corollary (1.12) are satisfied and the mapping $H$ has a coupled fixed point that is a solution in $M=C([a, b], R)$ of the integral equation (3).

\section{REFERENCES}

Agarwal, R.P., Hussain, N., \& Taoudi, M.A. 2012. Fixed point theorems in ordered Banach spaces and applications to nonlinear integral equations. Abstr. Appl. Anal., . Article ID 245872, 15 pages.

Akkouchi, M. 2011. Common fixed point theorems for two selfmappings of a b-metric space under an implicit relation. Hacet. J. Math. Statist., 40(6), pp. 805-810.

Aydi, H., Bota, M., Karapinar, E., \& Mitrović, S. 2012. A fixed point theorem for set-valued quasi-contractions in b-metric spaces. Fixed Point Theory Appl., 88.

Ansari, A.H., Chandok, S., \& Ionescu, C. 2014. Fixed point theorems on b-metric spaces for weak contractions with auxiliary functions. J. Inequal. Appl., 429.

Aghajani, A., Abbas, M., \& Roshan, J.R. 2014. Common fixed point of generalized weak contractive mappings in partially ordered b-metric spaces. Math. Slov., 4, pp. 941-960.

Bhaskar, T.G., \& Lakshmikantham, V. 2006. Fixed point theorems in partially ordered metric spaces and applications. Nonlinear Anal., 65, pp. 1379-1393.

Boriceanu, M. 2009. Strict fixed point theorems for multivalued operators in b-metric spaces. Int. J. Mod. Math., 4(3), pp. 285-301.
Boriceanu, M., Bota, M., \& Petrusel, A. 2010. Multivalued fractals in b-metric spaces. Cent. Eur. J. Math., 8(2), pp. 367377.

Bota, M., Molnar, A., \& Varga, C. 2011. On Ekelandsvariational principle in b-metric spaces. Fixed Point Theory, 12(2), pp. 21-28.

Bakhtin, I.A. 1989. The contraction mapping principle in quasimetric spaces. Funct. Anal., Unianowsk Gos. Ped. Inst., 30, pp. 26-37.

Czerwik, S. 1993. Contraction mappings in b-metric spaces. Acta. Math. Inform. Univ. Ostraviensis, 1, pp. 5-11.

Czerwik, S. 1998. Nonlinear set-valued contraction mappings in b-metric spaces. Atti. Sem. Mat. Fis. Univ. Modena, 46, pp. 263-276.

Guo, D., \& Lakshmikantham, V. 1987. Coupled fixed points of nonlinear operators with applications. Nonlinear Anal., 11, pp. 623-632.

Hussain, N., Abbas, M., Azam, A., \& Ahmad, J. 2014. Coupled coincidence point results for a generalized compatible pair with applications. Fixed Point Theory and Applications, 62.

Hussain, N., \& Shah, M.H. 2011. KKM mapping in cone bmetric spaces. Comput. Math. Appl., 62, pp. 1677-1684.

Huang, H., Paunović, Lj., \& Radenović, S. 2015. On some new fixed point results for rational Geraghty contractive mappings in ordered b-metric spaces. J. Nonlinear Sci. Appl., 8, pp. 800-807.

Luong, N.V., \& Thuan, N.X. 2011. Coupled fixed point in partially ordered metric spaces and applications. Nonlinear Anal., 74, pp. 983-992.

Mustafa, Z., Roshan, J.R., Parvaneh, V., \& Kadelburg, Z. 2013. Some common fixed point results in ordered partial b-metric spaces. J. Inequal. Appl., 562.

Mustafa, Z., Roshan, J.R., Parvaneh, V., \& Kadelburg, Z. 2014. Fixed point theorems for Weakly T-Chatterjea and weakly $\mathrm{T}$ Kannan contractions in b-metric spaces. J. Inequal. Appl., 46.

Mustafa, Z., Jaradat, M.M.M., Ansari, A.H., Popović, B.Z., \& Jaradat, H.M. 2016. C-class functions with new approach on coincidence point results for generalized [Formula: see text]weakly contractions in ordered b-metric spaces. Springerplus, 5(1), p. 802. pmid:27390643

Nieto, J.J., \& Rodríguez-López, R. 2005. Contractive Mapping Theorems in Partially Ordered Sets and Applications to Ordinary Differential Equations. Order, 22(3), pp. 223-239. doi:10.1007/s11083-005-9018-5

Nieto, J.J., \& Rodríguez-López, R. 2007. Existence and Uniqueness of Fixed Point in Partially Ordered Sets and Applications to Ordinary Differential Equations. Acta Mathematica Sinica, English Series, 23(12), pp. 2205-2212. doi:10.1007/s10114-005-0769-0

Olatinwo, M.O. 2008. Some results on multi-valued weakly jungck mappings in b-metric space. Central European Journal of Mathematics, 6(4), pp. 610-621. doi:10.2478/s11533-0080047-3

Wu, Y. 2008. New fixed point theorems and applications of mixed monotone operator. J. Math. Anal. Appl., 341, pp. 883-893. 